Research Article

\title{
Application of Internet of Things Sensor in Intelligent Art- Aided Design
}

\author{
Yihuai Xie $\mathbb{D}^{1}$ and Wei Wang $\mathbb{D}^{2}$ \\ ${ }^{1}$ College of Art and Design, Hunan First Normal University, Changsha, Hunan 410205, China \\ ${ }^{2}$ College of Arts and Crafts, Hunan Arts and Crafts Vocational College, Yiyang, Hunan 413000, China \\ Correspondence should be addressed to Yihuai Xie; xieyihuai@hnfnu.edu.cn
}

Received 12 August 2021; Revised 27 August 2021; Accepted 27 August 2021; Published 13 September 2021

Academic Editor: Guolong Shi

Copyright (C) 2021 Yihuai Xie and Wei Wang. This is an open access article distributed under the Creative Commons Attribution License, which permits unrestricted use, distribution, and reproduction in any medium, provided the original work is properly cited.

\begin{abstract}
As a new and popular technology, the IoT is deeply affecting the design method of art. It not only brings many benefits but also brings challenges to the field of information storage. The structure of intelligent art-aided design system based on IoT sensor is designed, and the traditional $\mathrm{C} / \mathrm{S}$ is applied in the application structure. The IoT information storage center needs to design a suitable data storage scheme according to its data characteristics. On the basis of fully mastering the principles and concepts of decorative art and the basic process of art-aided design, according to the magnanimity and temporal and spatial correlation, the application of this data distribution method to art-aided design can significantly improve the efficiency of art design. Experiments show that compared with similar random and Bubba algorithms, this strategy has better performance in system in terms of DM and LBST index. Finally, using the results of the control group and the experimental group, this paper verifies that the art-aided design system of the Internet of things can improve users' design enthusiasm; develop design potential and initiative; improve designers' observation ability, thinking method, and expression ability; and significantly improve users' design ability and design level.
\end{abstract}

\section{Introduction}

The creative design industry is heating up rapidly along with society development [1]. The continuous refinement of its functions and the continuous expansion of its application fields have triggered the rapid growth of demand for design talents. Art design covers a wide range, which can be divided into visual communication design, environmental art design, dyeing and weaving clothing design, industrial design, animation design, etc., and thus crossderived new professional fields, such as display design and jewellery design $[2,3]$.

The development of contemporary design and the future of design are determined by the degree of the combination of art and science, which can be seen from the development trend of contemporary design [4]. Taking information design as an example, information design is a new professional theory in the design discipline. At first, it mainly refers to the so-called interface design to solve the interface design problem between people and electronic products
[5]. In fact, the scope of information design is very wide. There are many topics of information design in many design fields, including graphic design. In a fundamental sense, information design is to express, convey, collect, and process information in the way and form of artistic design or provide a product or tool for people to use, understand, and obtain information. Software designers use numbers to process information, while art designers use art forms to express and process information through art design. Take posters in graphic design as an example. With the expansion of information dissemination in the information society, posters are required to convey more information. Designers use computers to express and convey a large amount of information in artistic forms [6].

Art design is more important to apply scientific theories and methods to traditional art design, so that modern art composition design presents a new way of existence and form [7]. This new way of existence and form is not a replacement, but a way to open up a new living space and 
build a new field outside the existing art ways and forms. This not only enriches the existing form of the original art but also changes the structural form of art in a new way of creation [8]. Although art designers do not realize the great difference between the generation and acquisition of images in information space and traditional manual drawing when using computers and programs to make images and design works, in fact, the generation and existence of virtual mode, the intermediary way to create and generate images in information space, are of great significance to mankind. Using computer-aided art to form design can not only regard computer as an advanced tool but also understand and explore the connotation of art design through surface phenomena [9].

The IoT is a new network technology based on sensor technology [10]. It is a network that combines all items with devices to realize data acquisition, fusion, and processing and intelligent identification and management through operation terminals. Literature [11] shows that the storage of IOT information should adopt distributed database to store the information centrally. The existing research on Internet of things data storage mostly focuses on the objectives and requirements of information center [12]. Literature [13] focuses on the objectives of Internet of things storage. In the initial stage of data distribution research, it mainly summarizes the problems and puts forward general solutions [14-16]. The most common algorithms are random algorithm, polling algorithm, and the famous bubble algorithm [17]. In recent years, according to the data characteristics and some distributed architectures, researchers have put forward targeted data distribution schemes. Literature [6] expounds the ultimate goal of data distribution in the sensor center and puts forward some framework suggestions, and the data center is established on the computer cluster. Literature [18] is a data allocation strategy for database clusters in main memory. The strategy measures the node load according to the access frequency and response time and incrementally copies the redundant data to each node. When the load is too heavy, each node will delete the redundant data with the least access in the node; this method has achieved good results in practice, but redundant backup will increase the communication overhead [19]. The strategy in literature [20] is to determine the data allocation strategy according to the data attribute information and store the highly correlated data in the same physical node, so as to minimize the $\mathrm{I} / \mathrm{O}$ overhead.

As an aided design tool, computer has entered the space of every designer with an irresistible force, and the development of computer art design covers all fields of practical art and pure art. This paper applies Internet of things technology to art-aided design system and designs the framework of $\mathrm{C} / \mathrm{S}$ and $\mathrm{B} / \mathrm{S}$. In order to improve the system efficiency, a data allocation algorithm of Internet of things based on dynamic storage is proposed. The experimental part verifies the reliability of the Internet of things data distribution algorithm based on dynamic storage. In addition, compared with the traditional control group, it is found that the art design system designed in this paper can significantly improve the art design level and reduce the design difficulty. The main contributions are summarized as follows: (1) this paper designs an intelligent art-aided design system based on $\mathrm{C} / \mathrm{S}$ mode and $\mathrm{B} / \mathrm{S}$ mode, which brings convenience to designers; (2) a data allocation algorithm of Internet of things based on dynamic storage to improve the system efficiency; and (3) experiments are designed and compared with the control group to verify the superiority of the art assistance system.

\section{The Structure Design of Art-Aided Design System Based on IoT}

2.1. Art-Aided Design System Based on Internet of Things. In art-aided design system based on IoT sensor [21], the source of art-aided design data is different from the sensor of the IoT. The data processing system needs special sensing equipment to collect and preprocess the data. The preprocessed data needs to be aggregated through the network, and the aggregated data can be used as the source data for big data processing and analysis after reprocessing. Therefore, for the generation and preprocessing mechanism of art design big data, the equipment for collecting, transmitting, and preprocessing negative and expensive data must be designed as a separate layer. In this layer, the initial energy consumption data is collected, transmitted, preprocessed, and finally delivered to the upper layer. At the same time, the design of this layer should try to achieve low coupling between different modules, so that for different art-aided design environments, it can be applied to new scenes only by slightly replacing modules such as acquisition nodes. Secondly, when the underlying sensing data is preprocessed and forwarded, it should be received and stored by the system layer for big data-related processing. In this layer, relevant big data processing tools are used to process massive data to meet the needs of user applications, such as data query, data analysis, and data mining. This layer also needs to complete data storage and task scheduling and can support mainstream big data processing functions as much as possible. Therefore, the art-aided design system is composed of three parts: wireless sensor network, data transmission, and application design layer.

(1) Wireless sensor network infrastructure includes sensor nodes and wireless gateways. The wireless nodes and gateway devices in this layer are uniformly managed by the server. The main functions of the server include processing new wireless node network access requests and processing and storing sensor data. At the same time, the real-time display function code of wireless sensor network data will also be deployed in the server of the sensor network layer. The sensor network layer will provide the original data for the upper platform and applications. The real-time display function of the sensor network layer data is also deployed in this layer, and the function of importing the sensor data into the distributed storage system is completed. In the whole big art-aided design platform, the sensor network layer plays a basic supporting role; (2) the transmission layer mainly applies the mainstream data transmission technology obtained by the underlying wireless network to the art-aided design system through the network. Mainstream data transmission technologies include Bluetooth, $3 / 4 \mathrm{G}$, 


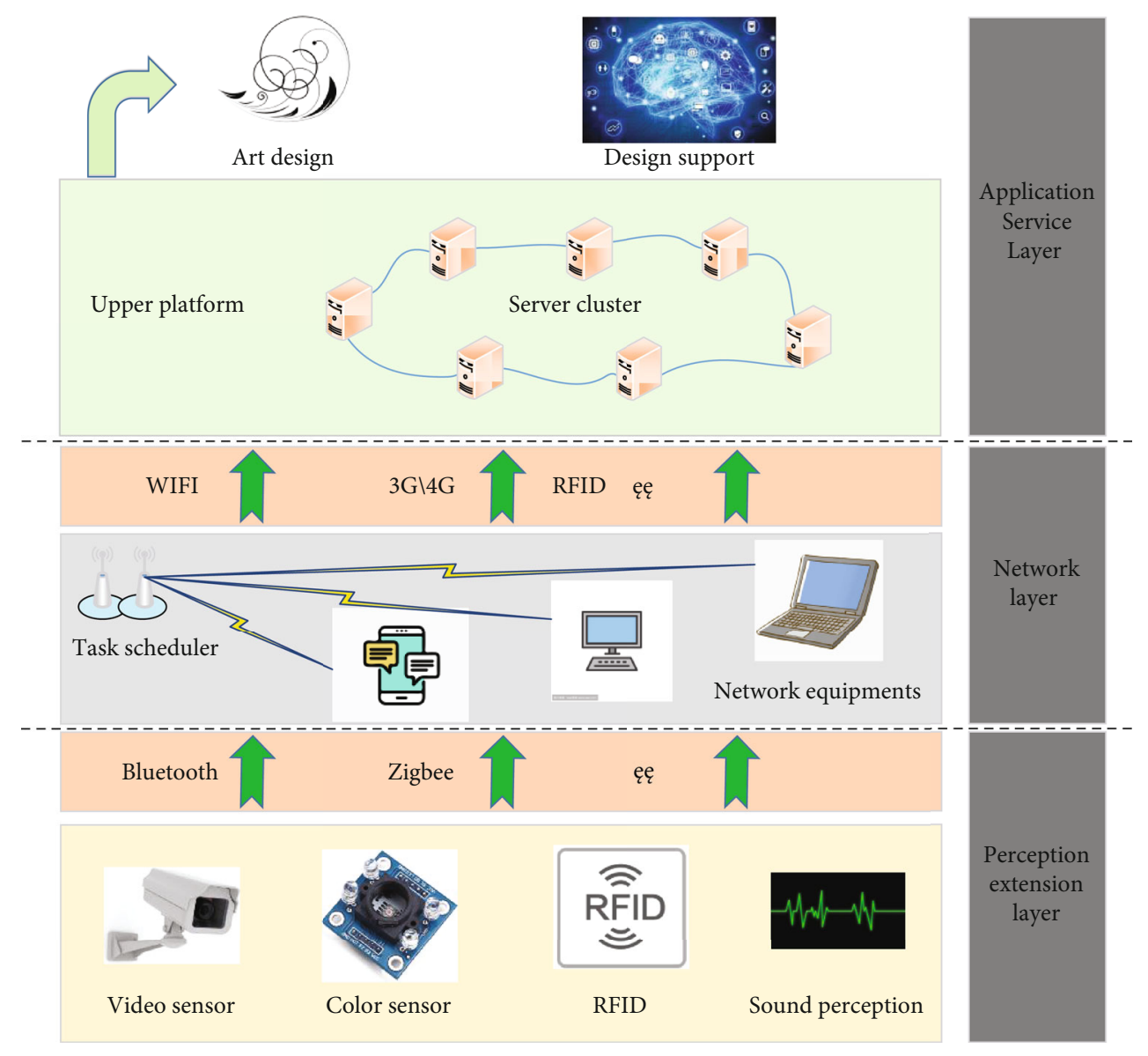

FIGURE 1: The architecture of the art-aided design system based on IoT.

and WiFi technologies; (3) application service layer: as an aided design tool, computer has entered the space of every designer with an irresistible force, and the development of computer art design covers all fields of practical art and pure art. Artists and art designers are also increasingly using computers to assist creation in their work. The digital operation of art has changed the traditional hand-painted method and brought great changes to the design and creation process. The wonderful colours and unpredictable shapes displayed by art-aided design are realized through the foundation of the underlying sensor network (Figure 1).

\subsection{The Configuration of Art Design Service System. This} paper adopts three-tier C/S mode; the framework of intelligent art-aided design system based on C/S mode is shown in Figure 2. The client is useful for realizing the interface function and encapsulating part or all of the application logic. The database server on the server side mainly provides data. It is mainly divided into client, server, and middleware. Among them, (1) the client application layer usually implements the user interface, which provides a visual interface to display information and collect data. It only deals with the application server. (2) Middleware usually implements application logic and is a bridge between customers and database servers. It responds to the requests sent by Sichuan users, performs some business tasks, and deals with the data- base server. In the process of practical application, the components of this layer can usually be divided into more than two levels, so this structure is also called multilevel structure. (3) The server management program (server) realizes the definition, maintenance, access, update, and management of data and responds to the data request of the application server. Its physical implementation can be in a database management system or a collection of multiple heterogeneous databases, which can reside on a variety of platforms.

The application server can control which data is changed and accessed, as well as the data change and access mode. In addition, it can control the storage permissions of the application. In this way, if another security mechanism is provided in the application server and database server. In the three-tier $\mathrm{C} / \mathrm{S}$ structure, the settings or drivers required to deal with the database server are borne by the application service and make the database server focus on data services rather than frequent communication with client applications. The logical relationship of each layer is clear, and the "thin customer" is really achieved.

When the number of customers increases, the system load is too large, the operation is slow and even crashes. On the client side, when there are many customers, the system installation and upgrade are inconvenient. In order to solve the excessive "bloating" of the client or server, the* layer and (mode came into being. As shown in Figure 2, 


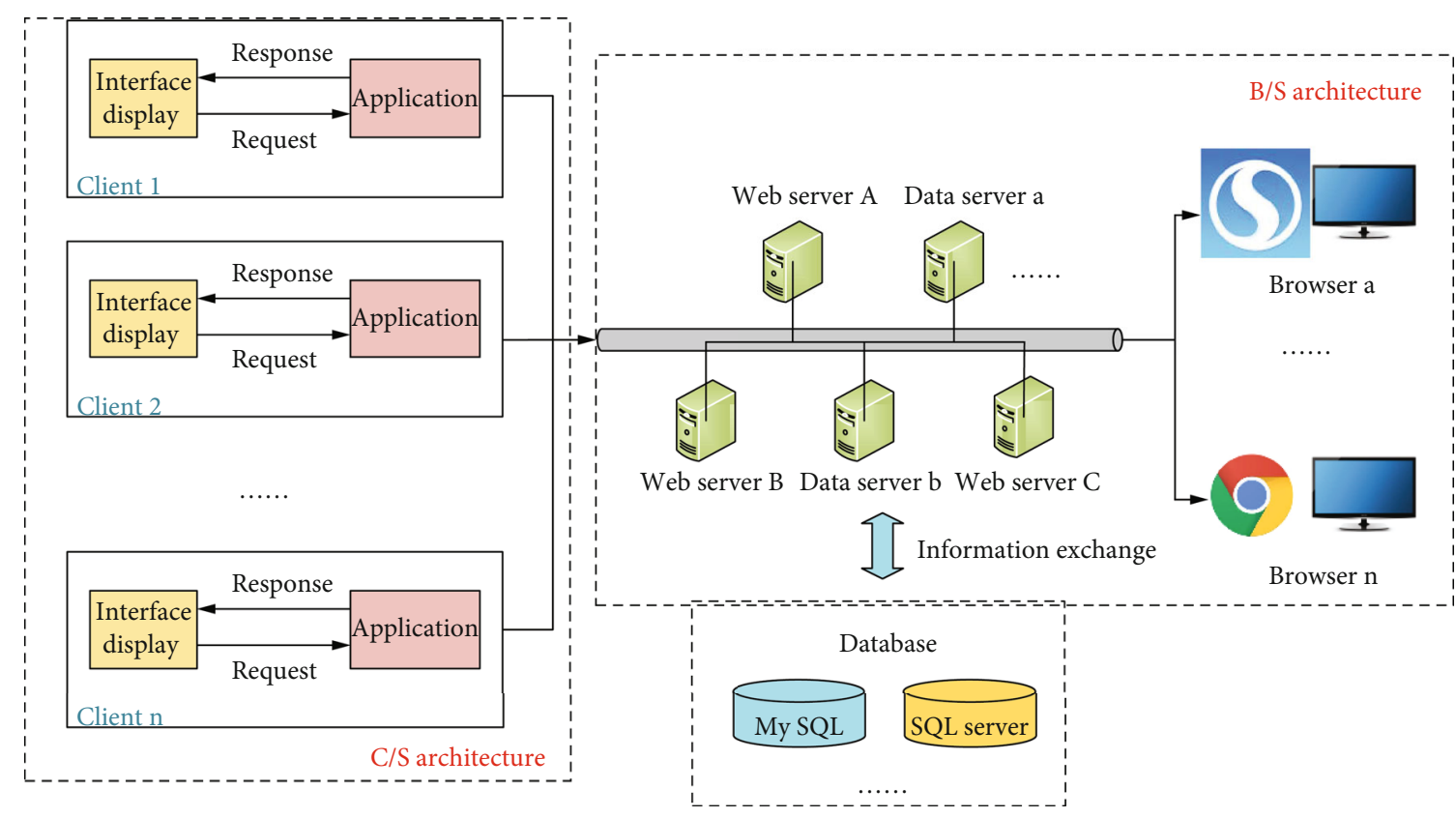

FIGURE 2: Framework of intelligent art-aided design system based on C/S mode.

an application server (middle layer) was added between the client and the server, and the system business logic was placed on this layer, which was completely separated from the client and the database. The deletion of business logic would not affect the client and the database server.

\section{Research on Real-Time Data Distribution Strategy for Sensor Information of IoT}

3.1. Framework Diagram of Distributed Real-Time Database System. The process of data allocation is to send the file slices generated after data redundancy to each storage node for storage. Because the performance of different storage nodes is different, different allocation schemes will have different effects on the performance index of distributed system. The purpose of data allocation is to make the overall performance indicators of the distributed system meet the needs of users. At present, the commonly used data allocation methods can be divided into three kinds: graph theory, mathematical programming, and heuristic algorithm. In graph theory, the most important method applied to data distribution is the matching algorithm of bipartite graph. The whole system is described as a bipartite graph, in which there are two sets, namely, data set and node set, and the connection between data and nodes is used to represent the distribution mode of data. This method is simple and intuitive, but it also has its limitations. It can only be applied when the number of data and nodes is small and cannot be applied to practical engineering.

Figure 3 shows the dynamic data distribution system framework adopted by ATDA strategy. The first system generates a first registration information table. The first system also assigns a unique category number to the Internet of things device. The second system generates a second registration information table and a communication object. Sec- ond, the system also creates a policy management object. The policy management object also establishes a linkage processing registration form, authenticating and verifying the communication object in the second system with the Internet of things device. The communication equipment in the second system communicates with the Internet of things equipment and obtains the state and data of the Internet of things equipment in real time, and the strategy management object processes the obtained state and data. The beneficial effects of the invention are that the Internet of things equipment can realize intelligent management locally and realize the linkage of things, and the data can be stored locally and transmitted remotely; it effectively improves the overall efficiency of the communication system and gives full play to the role of the Internet of things.

\subsection{Design for Dynamic Data Distribution (ATDA) Scheme.} The dynamic data distribution (ATDA) scheme proposed in this paper can be expressed as follows: data allocation is the process of mapping data blocks to each storage node. A mathematical model can be established to analyse it. The input is the set of data blocks, and the output is the division of the set of data blocks. The mapping process can be defined as a transfer matrix, which maps the input to the output according to a certain objective function constraint.

Definition 1. The input vector of the data model is as follows: Blob $=\left[\text { blob }^{1}, \text { blob }^{2}, \mathrm{~L}, \mathrm{blob}^{n}\right]^{T}$ where blob represents the data block.

Definition 2. The output vector y of the data model $Y=\left[y_{1}\right.$, $\left.y_{2}, \cdots, y_{n}\right]$, where component $y_{i}$ represents the load rate of each physical node. 


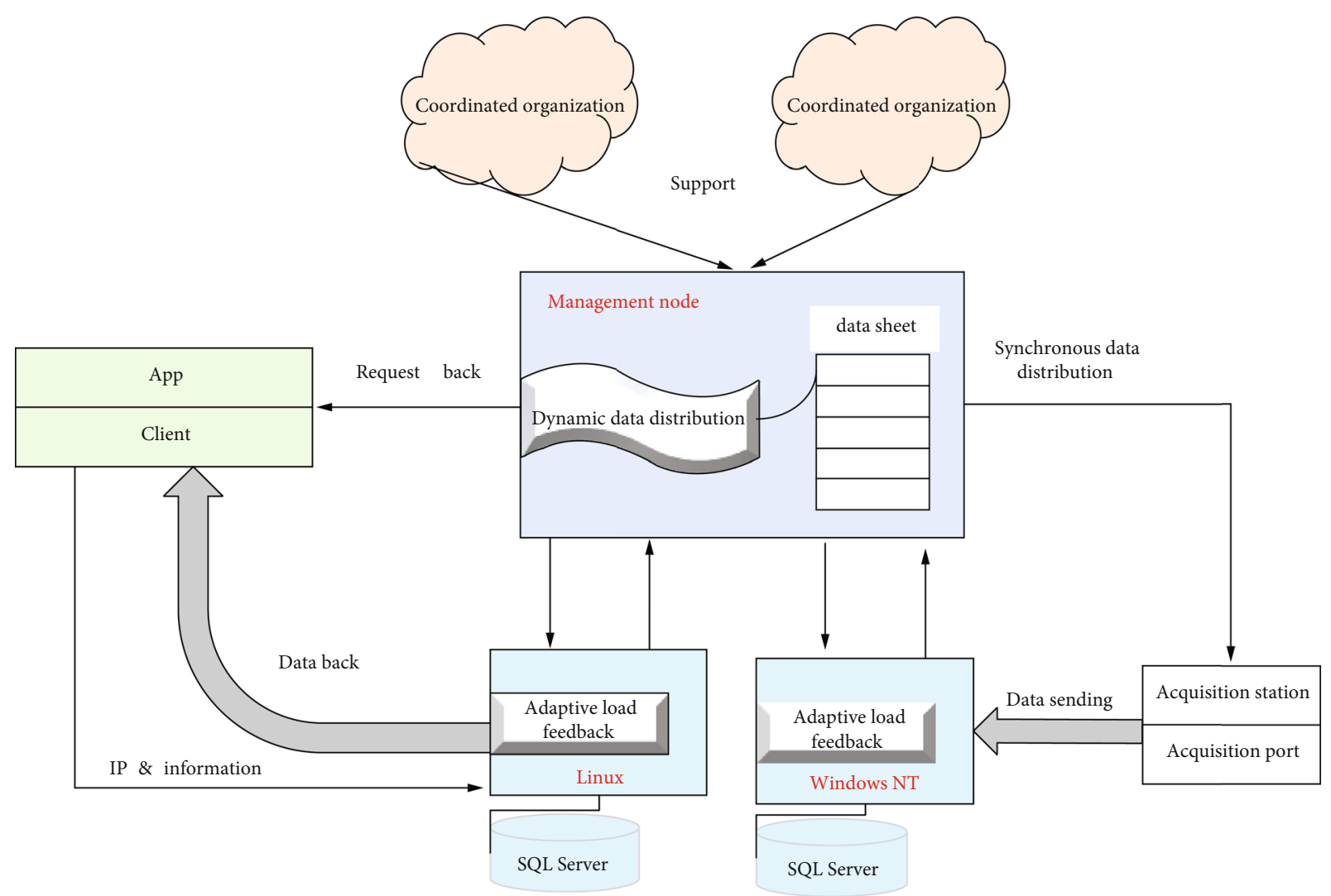

Figure 3: Dynamic data distribution model of Internet of things.

Definition 3. The transfer matrix of the data model is $\mathbf{X}$, and it meets $\sum x_{i j}=1$. Therefore,

$$
\mathbf{Y}=\mathbf{B l o b} \times \mathbf{X}
$$

where $\mathbf{B l o b}$ is the input vector of the data model and $\mathbf{X}$ is transfer matrix.

According to the above mathematical model, the static allocation strategy initializes and allocates the data block to the storage node by using the spatial correlation of sensor information. In practical application, the data block exists in the form of data points, and each data point supports all required data types, and each data point occupies the same memory space. Therefore, the input vector blob can be simplified as $\operatorname{tag}=\left[\operatorname{tag}_{1}, \cdots, \operatorname{tag}_{m}\right]^{T}$. The load brought by the data block to the physical node in the time domain is dynamic, so the data module needs to be considered in the time domain. When the vector tag is considered in the fixed time domain length $t, \operatorname{tag}=\left[\operatorname{tag}_{1}, \cdots, \operatorname{tag}_{m}\right]^{T}$. Since the sensor data sampling represents the continuously changing state with discrete values, the update of data points is also discrete, and Poisson distribution is used to simulate the probability distribution of random arrival of discrete events. Therefore, maximum load vector of $n$ physical nodes

$$
\operatorname{node}_{\max }=\left[\operatorname{node}_{(1, \max )}, \cdots, \operatorname{node}_{(n, \max )}\right] .
$$

Storage node unit load rate vector is calculated as

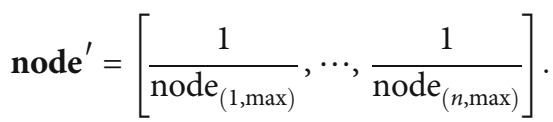

Then, the static allocation model of data is as follows:

$$
\begin{aligned}
\mathbf{Y}^{\prime} & =\left[y_{1}^{\prime}, \cdots, y_{n}^{\prime}\right]=\text { node }_{\max } \times \mathbf{X} \times \mathbf{B}(\lambda)^{T} \\
& =\left\{\begin{array}{l}
\operatorname{diag}\left[\frac{1}{\operatorname{node}_{(1, \max )}}, \cdots, \frac{1}{\operatorname{node}_{(n, \max )}}\right] \times X \times B(\lambda)^{T}, \\
\sum x_{i j}=1,
\end{array}\right.
\end{aligned}
$$

where each component of the output vector $\mathbf{Y}^{\prime}$ represents the load rate of each physical node with a time domain length of $t$. it is a vector composed of random variables. Considering the load balance, it is required to minimize the variance between the components of the output vector.

Therefore, the basic idea of setting the storage node overload threshold in this algorithm is shown in Figure 4. As shown in Figure 4, this paper designs a threshold-based Internet of things node data allocation algorithm. When the node data is higher than the threshold, it moves to the low node load, and vice versa. At this time, the quality of the Internet of things data distribution strategy directly 


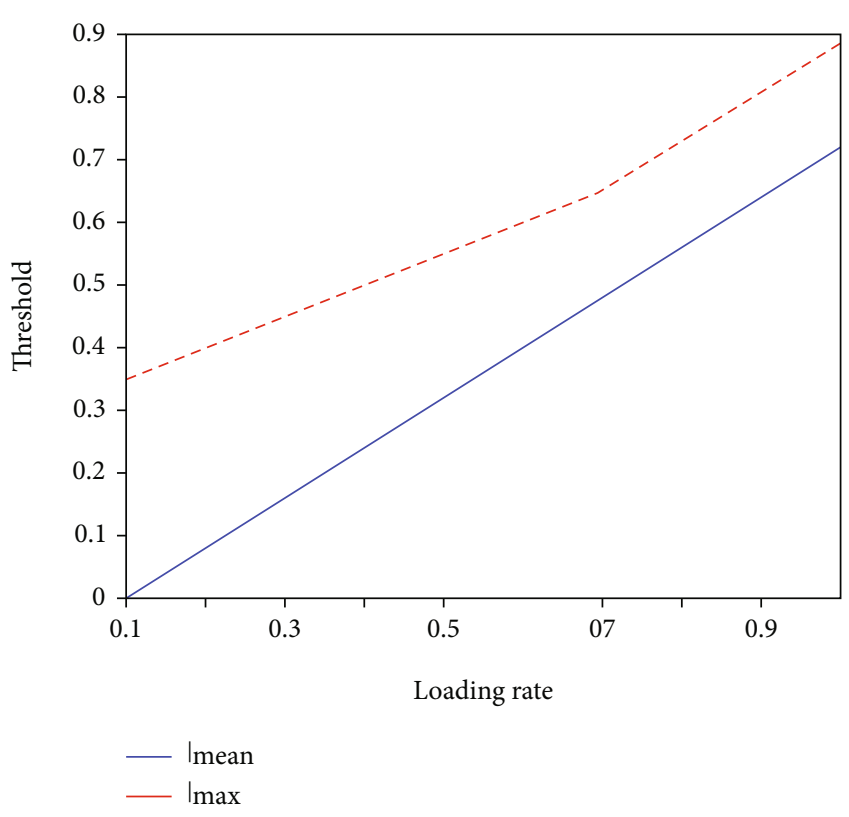

FIGURE 4: Load threshold figure for the ATDA strategy.

affects the perception effect of the network on the physical quantity of the monitoring environment. A good control strategy is the prerequisite to ensure that the network collects complete and accurate data from the physical world, and it is the first step to realize the tasks performed by all sensor networks. Wireless sensor network nodes are deployed in the monitoring area through high density to ensure the high coverage of the network and improve the reliability of the network. Therefore, there are many redundant nodes, resulting in a large amount of data redundancy in the process of information transmission, which increases the network energy consumption and reduces the network life. The network coverage control strategy through node scheduling, on the premise of ensuring the coverage, makes some nodes sleep and reduces the number of nodes working at the same time, which not only reduces the unnecessary energy consumption caused by redundant information communication but also increases the average life of nodes in the whole network

The load threshold heuristic function is defined as follows:

$$
l_{\text {max }}= \begin{cases}\alpha \ln \left(e+\varepsilon \times\left(l_{\text {mean }}-\alpha\right)\right)+\beta & l_{\text {mean }} \leq \alpha \\ l_{\text {mean }}+\beta & l_{\text {mean }}>\alpha\end{cases}
$$

where $l_{\max }$ represents the overload threshold of storage nodes, $l_{\text {mean }}$ represents the average load, and $\alpha$ is threshold for measuring severe overload. The execution flow chart of the algorithm is shown in Figure 5.

The art-aided design system provides a command line interface, which is suitable for remote login to the host to query, as shown in Figure 5. The search operation is carried out through the client page in the art-aided design system. Similarly, the middleware layer will forward and filter the commands of the client, which not only ensures the ease of

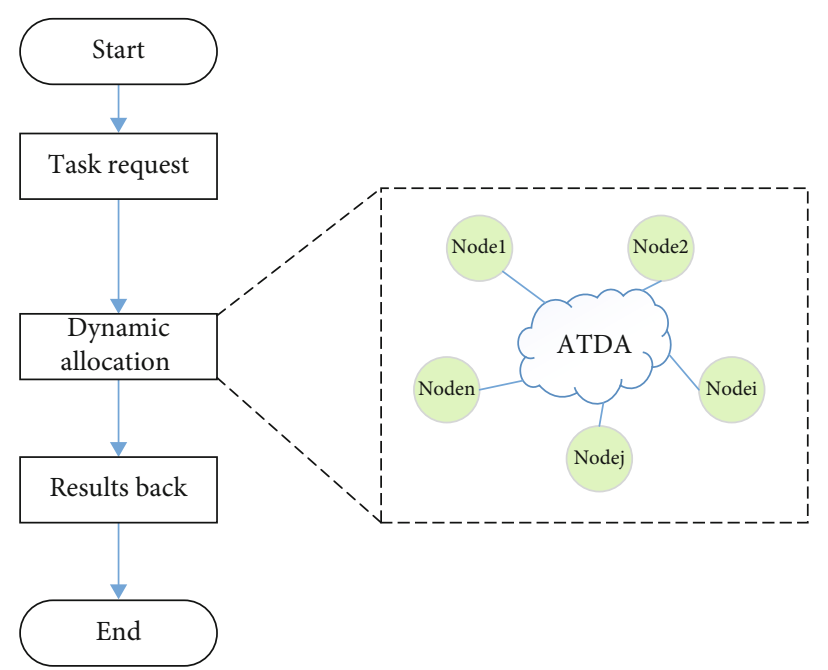

FIgURE 5: Load threshold figure for the ATDA strategy.

use of the query function but also solves the security problem of the system. When the shape request is initiated, the system will directly call the data of the storage node and adopt the dynamic data allocation algorithm to improve the search efficiency. After the search data is returned, the user can select the appropriate design elements.

\section{Simulation Results and Performance Analysis}

4.1. Experimental Environment and Parameter Setting. In the experimental part, we mainly carried out two parts of comparative experiments: (1) compared with the traditional data allocation algorithm of the Internet of things system, we designed DM index, LBST index, and LBOT index to verify the advantages of the dynamic data allocation algorithm proposed in this paper; (2) organized the experiment and used the traditional art design system as the control group. The art-aided design system designed in this paper is used as the experimental group. 30 designers with the same basis of art design are selected as the experiment. 15 people are randomly selected as the control group, and the other 15 as the experimental group.

This experiment is run on the real-time IoT data system, the comparison algorithms used in the experiment are Bubba and random algorithm. Random is the most common data allocation strategy. Although Bubba was proposed as early as 1988, it can improve the system response time. Google learned from Bubba's ideas to improve its memory and disk performance; the short-term domain load balancing and load migration of the system are compared with Bubba and random algorithms. In addition, the intelligent art-aided design based on Internet of things sensor is compared with the traditional art-aided design system to verify the superiority of this system.

For the experimental environment, the number of storage nodes is 10 , the disk capacity of each storage node ranges from $100 \mathrm{~GB}$ to $1 \mathrm{~TB}$, the memory ranges from $1 \mathrm{~GB}$ to $4 \mathrm{~GB}$, and the CPU is Intel Xeon CPU x3430 2.40 GHz, which can 


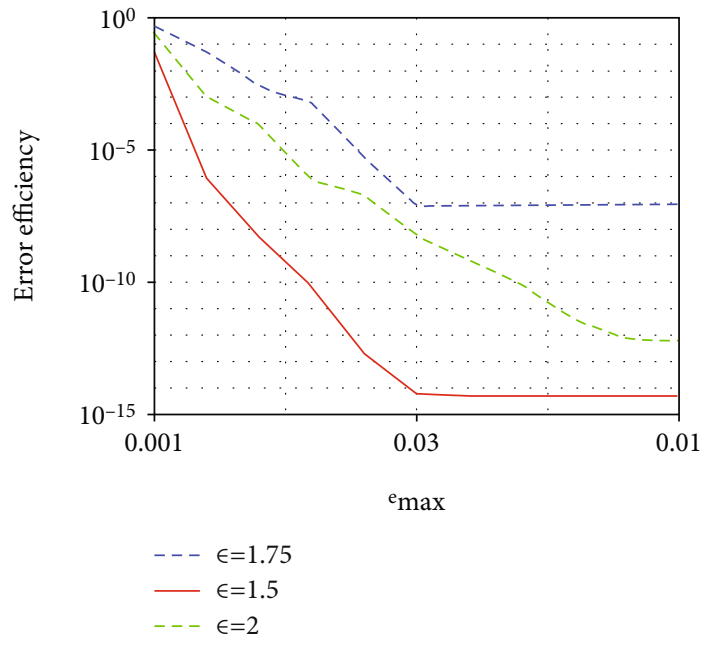

(a) Parameter selection for $\varepsilon$

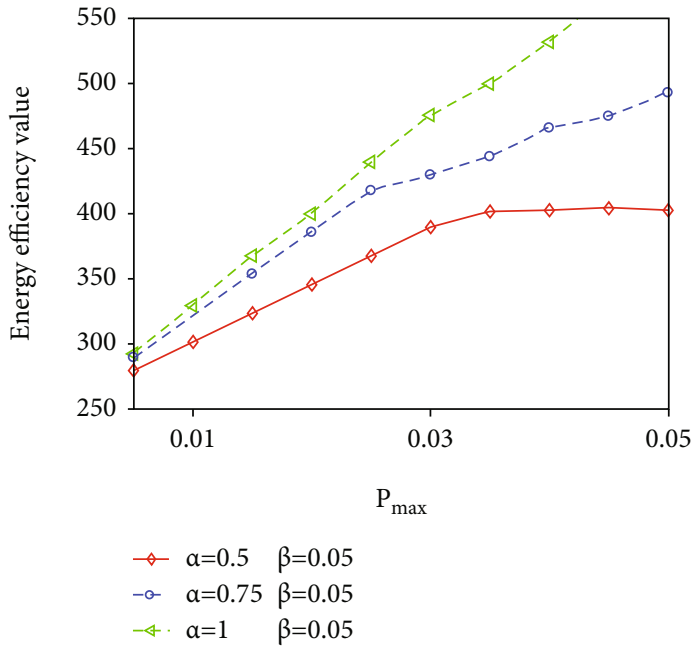

(b) Parameter selection for $\alpha, \beta$

FIgURE 6: Three-axis acceleration collection results for the elderly at home.

accommodate the maximum load data points ranging from 100000 to 400000 .

For the initialization parameters, the parameters in the ATDA strategy are set as follows: overload threshold parameters $\alpha=0.75$, overload threshold offset $\beta=0.05$, and curvature parameter according to $\in\left[1,1 / \alpha^{2}\right]$. The requirement of 2 is set to $\varepsilon=1.7$. In the adaptive time-domain feedback algorithm, the initial feedback time domain is set to $10 \mathrm{~s}$ and the time domain threshold is set to $1 \mathrm{~s} \sim 100 \mathrm{~s}$. The comparison algorithm Bubba stores data points in a nonredundant way. The programming language is $\mathrm{C}++$.

4.2. Parameter Selection for the ATDA Strategy. In order to select the optimal parameters, the following comparative experiments are designed to achieve the optimal data distribution. The simulation results are shown in Figure 6.

Figure 6(a) is a simulation of different channel estimation error values under the imperfect channel between the secondary system and the primary system. Because this paper considers the bounded uncertainty model for this model, for different estimation error values $e_{\max }$, this paper needs to confirm which error value has influence on the overall energy. For the imperfect channels between secondary systems, for the parameter values in them, different parameters can be obtained through simulation $\sigma$ influence and $e_{\max }$ values on energy efficiency. It can be seen from Figure 6(a) that with the increase of the channel estimation error value $e_{\max }$ between the primary system and the secondary system, the energy efficiency will decrease; when there is imperfect channel correlation coefficient between the secondary IOT user equipment and the secondary base station $\varepsilon$, the maximum energy efficiency value is obtained. It can be concluded that in the imperfect channel model, the best parameter matching between the primary system and the secondary system, the secondary user and the secondary base station are $e_{\max }=0.001$. Figure 6(b) shows different weighting factors obtained in the weighted method $\alpha, \beta$. The value varies with the maximum transmission power $P_{\max }$ allowed by each secondary IOT device user. As is shown, when the maximum allowable user transmission power increases, the energy efficiency increases accordingly. With the increase of $P_{\max }$, it shows that the power range available to each secondary IOT device user becomes larger, thus increasing the energy efficiency value. As can be seen from equation (5), $\alpha$ is the weight of the total transmission rate. As the value of $\alpha$ increases, the total transmission rate also increases. When $P_{\max }$ reaches $0.6 \mathrm{~W}$, the change trend of the three curves tends to slow down. When $\alpha=0.75, \beta$ $=0.05$, which means that all weights are added to the total transmission rate. At this time, the total data transmission rate increases linearly, and the energy efficiency value reaches the maximum.

4.3. Performance Verification of the ATDA Strategy. Figure 7 shows the simulation results of the proposed method, compared with Bubble [22-24] and Random algorithms [25]. As shown in Figures 7(a)-7(c), in the LBST index and DM index, the ATDA strategy is better than the Bubba algorithm and random algorithm. The LBST index is the mean of the system load variance in the short-term domain, which more accurately reflects the load balance of the real-time system.

The load imbalance in the short-term domain makes the load of some storage nodes too high in a short time, which will seriously affect the system efficiency and the performance index of the sensing information of the IoT. When the system is under low load, the results of the LBST index for our proposed method are similar to those of Bubba and random algorithms. The adaptive time-domain feedback algorithm in this paper changes the time interval of feedback load on the basis of the change of system load; the ATDA strategy tolerates large load fluctuations at low load but strictly limits load fluctuations at high load to achieve load balancing. At the same time, storage nodes adaptively scale the feedback time domain to ensure load balancing in the 


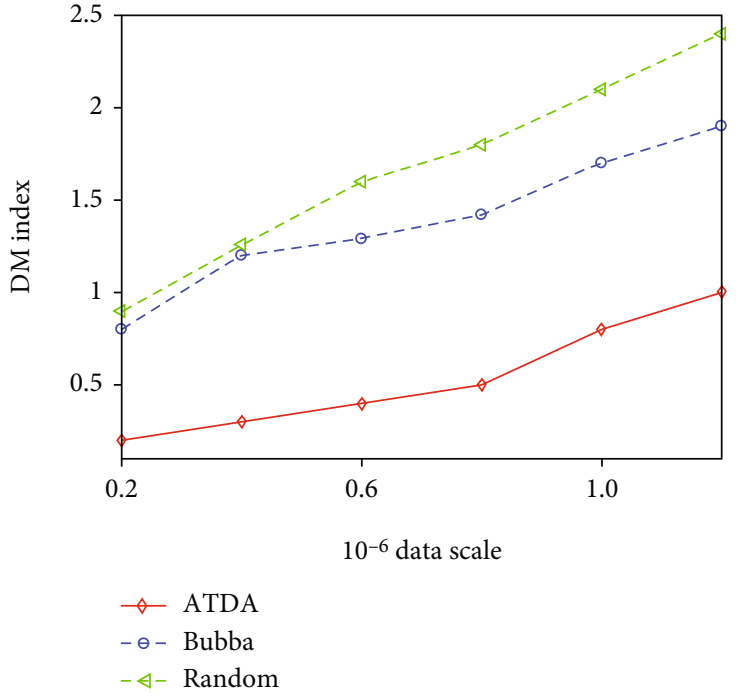

(a) DM index comparison

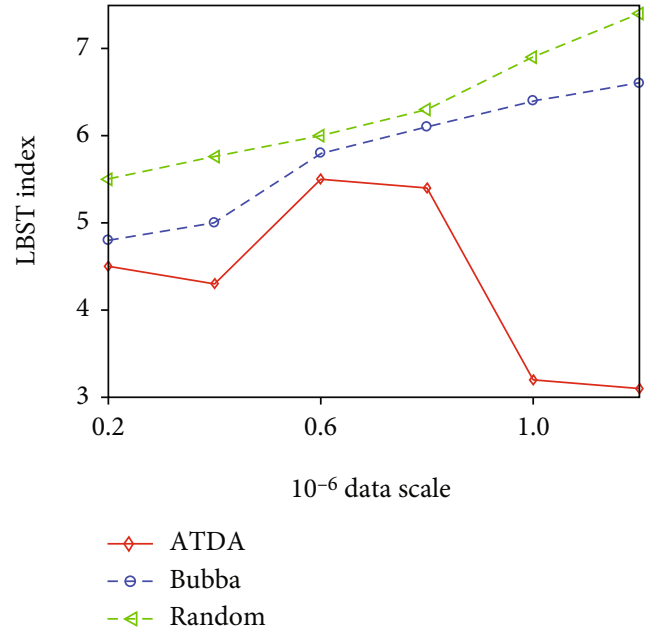

(b) LBST index comparison

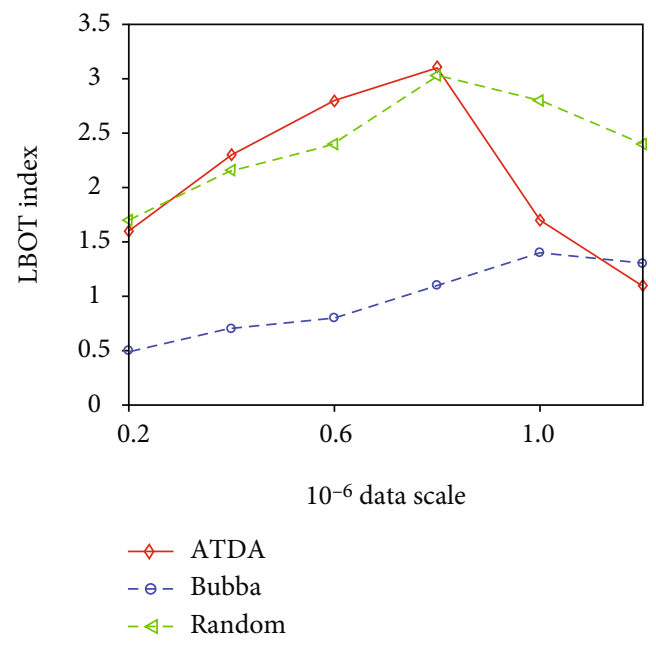

(c) LBOT index comparison

FIGURE 7: Three-axis acceleration collection results for the elderly at home.

short-term domain. The LBOT index measures the system load balance by describing the system variance of the termination state. Therefore, the algorithm proposed in this paper can improve the data allocation efficiency under heavy load and improve the service life of IoT data nodes.

\subsection{Analysis of Experimental Test Results for Art Design} Based on IoT. Practice is an important part of art-aided design system evaluation. To verify the advantages of Internet of things sensors in art-aided design, we must objectively evaluate the advantages and disadvantages of the platform through experiments. In this paper, 30 designers with the same basis of art design are selected as the experiment. 15 people are randomly selected as the control group, and the other 15 as the experimental group. It is compared and verified from three aspects: home decoration design, office space design, and business space design. The comparison of the control and experimental group is shown in Table 1 .
It can be seen from Table 1 that the users using the intelligent art-aided design system based on IoT sensors are better than those in the traditional control group. Especially in business space design, the passing and excellent proportion of the experimental group is better. In addition, the experimental group also has obvious advantages in home decoration design and office space design.

Art design emphasizes the reasonable connection of disciplines, strives to realize the overall education; takes the history, culture, and national background of art as the basis of art curriculum; and emphasizes the cultural diversity of art education. Modern art pursues diversity in forms, seeks novelty in visual effects, and integrates artistic methods with marginal disciplines, including design art, scientific and technological manufacturing, and commercial behaviour. Designers must have a certain accumulation of scientific and cultural knowledge in order to keep up with the development of art design. Therefore, this paper verifies the 
TABLE 1: The comparison of the control group and experimental group.

\begin{tabular}{|c|c|c|c|c|c|}
\hline & & Excellent & Medium & Pass & Fail \\
\hline \multirow{2}{*}{ Decoration design } & Control group & 2 & 4 & 4 & 5 \\
\hline & Experimental group & 4 & 5 & 3 & 3 \\
\hline \multirow{2}{*}{ Office design } & Control group & 1 & 3 & 6 & 5 \\
\hline & Experimental group & 3 & 5 & 4 & 3 \\
\hline \multirow{2}{*}{ Business design } & Control group & 3 & 3 & 3 & 6 \\
\hline & Experimental group & 5 & 4 & 4 & 2 \\
\hline
\end{tabular}

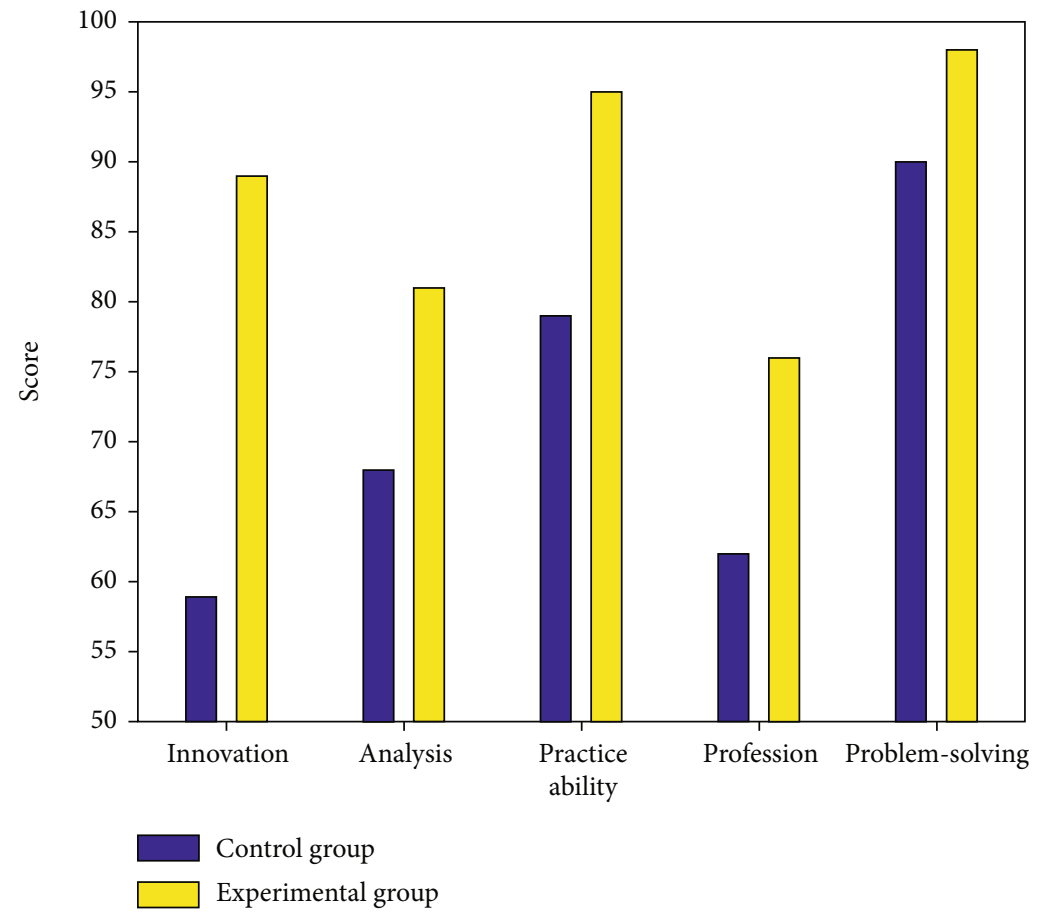

FIGURE 8: BP neural network heart disease recognition rate.

important role of IOT sensors in art-aided design from the perspectives of innovation ability, problem-solving ability, practical ability, and professional ability. The comparison results between the control group and the experimental group are shown in Figure 8.

From the comparison results in Figure 8, the average scores of each ability point have been improved. In particular, the average scores of innovation ability, analysis ability, problem-solving ability, practical ability, and professional ability have increased significantly.

\section{Conclusion}

In the computer technology integrated with art and design, scene visual understanding is one of the most widely used technologies in the field of art and design. Scene visual understanding is to use computer to simulate human visual function; use computer to replace human eyes and brain; perceive, recognize, and understand three-dimensional scenes and objects in the objective world; integrate with natural language; analyse complex object distribution problems in scene images; accurately describe the obtained information in a reasonable way; and help designers extract scene information data. The art-aided design method combined with visual scene solution algorithm can effectively help designers solve the problem of unclear output image due to inaccurate extracted scene image information data in art creation. Therefore, based on the application background of art-aided design, using the Internet of things system to extract the scene in the environment can accelerate the visual understanding of the applicable scene and simplify the design algorithm. Therefore, this paper designs an art design system based on the Internet of things. In order to achieve efficient art design, this paper designs an intelligent art-aided design system based on IoT sensors and designs a design element search framework based on $\mathrm{C} / \mathrm{S}$ mode. Experiments show that this strategy is outstanding. Compared with the traditional art-aided design system, it verifies 
the advantages of the art-aided design system designed in this paper. In the next work, we will further study the relevant optimization problems for the sensor information of the Internet of things, taking into account the query and update operation. Combined with the characteristics of sensor information and the advantages of distributed computing framework, we will more comprehensively and systematically study the data allocation optimization of sensor information of the Internet of things in the case of frequent queries.

\section{Data Availability}

The data used to support the findings of this study are available from the corresponding author upon request.

\section{Conflicts of Interest}

We declare that there is no conflict of interest.

\section{Acknowledgments}

The study was supported by "Philosophy and Social Science Foundation of Hunan Province Project, China (Grant No. 17YBA112)."

\section{References}

[1] K. Thongpull and A. König, "Advance and case studies of the DAICOX framework for automated design of multi-sensor intelligent measurement systems," tm-Technisches Messen, vol. 83, no. 4, pp. 234-243, 2016.

[2] L. Zhang, H. Yuan, S. H. Chang, and A. Lam, "Research on the overall architecture of Internet of Things middleware for intelligent industrial parks," The International Journal of Advanced Manufacturing Technology, vol. 107, no. 3-4, pp. 1081-1089, 2020.

[3] Y. Song, "Application of symmetry algorithm in energy optimal allocation of wireless sensor networks," Journal of Intelligent \& Fuzzy Systems, vol. 38, no. 6, pp. 7715-7724, 2020.

[4] M. Nkomo, G. P. Hancke, A. M. Abu-Mahfouz, S. Sinha, and A. J. Onumanyi, "Overlay virtualized wireless sensor networks for application in industrial internet of things: a review," Sensors, vol. 18, no. 10, p. 3215, 2018.

[5] J. K. Jensen, R. Skår, and B. Tveit, "Introducing the National Early Warning Score-a qualitative study of hospital nurses' perceptions and reactions," Nursing Open, vol. 6, no. 3, pp. 1067-1075, 2019.

[6] A. Venkatanarayanan, A. Vijayavel, A. Rajagopal, and P. Nagaradjane, "Design of sensor system for air pollution and human vital monitoring for connected cyclists," IET Communications, vol. 13, no. 19, pp. 3181-3186, 2019.

[7] L. Li, Y. Zheng, M. Yang et al., "A survey of feature modeling methods: historical evolution and new development," Robotics and Computer-Integrated Manufacturing, vol. 61, article 101851, 2020.

[8] T. Adegbija, A. Rogacs, C. Patel, and A. Gordon-Ross, "Microprocessor optimizations for the internet of things: a survey," IEEE Transactions on Computer-Aided Design of
Integrated Circuits and Systems, vol. 37, no. 1, pp. 7-20, 2018.

[9] Y. Liu, L. Zhang, Y. Yang et al., "A novel cloud-based framework for the elderly healthcare services using digital twin," IEEE Access, vol. 7, pp. 49088-49101, 2019.

[10] S. Zeadally and O. Bello, "Harnessing the power of Internet of Things based connectivity to improve healthcare," Internet of Things, vol. 14, article 100074, 2021.

[11] S. Kang, H. Baek, E. Jung, H. Hwang, and S. Yoo, "Survey on the demand for adoption of Internet of Things (IoT)-based services in hospitals: investigation of nurses' perception in a tertiary university hospital," Applied Nursing Research, vol. 47, pp. 18-23, 2019.

[12] Y. Qian, L. Shi, J. Li et al., "A workflow-aided Internet of things paradigm with intelligent edge computing," IEEE Network, vol. 34, no. 6, pp. 92-99, 2020.

[13] G. Cai, Y. Fang, P. Chen, G. Han, G. Cai, and Y. Song, "Design of an MISO-SWIPT-aided code-index modulated multicarrier M-DCSK system for e-health IoT," IEEE Journal on Selected Areas in Communications, vol. 39, no. 2, pp. 311324, 2021.

[14] O. Bello and S. Zeadally, "Intelligent device-to-device communication in the internet of things," IEEE Systems Journal, vol. 10, no. 3, pp. 1172-1182, 2016.

[15] F. Castaño, S. Strzelczak, A. Villalonga, R. E. Haber, and J. Kossakowska, "Sensor reliability in cyber-physical systems using internet-of-things data: a review and case study," Remote Sensing, vol. 11, no. 19, p. 2252, 2019.

[16] P. Panchatcharam and S. Vivekanandan, "Internet of things (IOT) in healthcare - smart health and surveillance, architectures, security analysis and data transfer," International Journal of Software Innovation (IJSI), vol. 7, no. 2, pp. 2140, 2019.

[17] I. de la Torre Díez, S. G. Alonso, S. Hamrioui, E. M. Cruz, L. M. Nozaleda, and M. A. Franco, "IoT-based services and applications for mental health in the literature," Journal of Medical Systems, vol. 43, no. 1, p. 11, 2019.

[18] J. Gan, X. Wang, J. Zhou, L. Tang, and L. Yuan, "Intelligent monitoring network construction based on the utilization of the Internet of Things (IoT) in the metallurgical coking process," Open Physics, vol. 16, no. 1, pp. 656-662, 2018.

[19] B. Wang, W. Kong, H. Guan, and N. N. Xiong, "Air quality forecasting based on gated recurrent long short term memory model in Internet of Things," IEEE Access, vol. 7, pp. 6952469534, 2019.

[20] C. Zhang and Z. Liu, "Application of big data technology in agricultural Internet of Things," International Journal of Distributed Sensor Networks, vol. 15, no. 10, 2019.

[21] Y. Wang, Y. Liu, C. Wang et al., "Storage-less and converterless photovoltaic energy harvesting with maximum power point tracking for internet of things," IEEE Transactions on Computer-Aided Design of Integrated Circuits and Systems, vol. 35, no. 2, pp. 173-186, 2016.

[22] M. M. Hussain, M. M. S. Beg, and M. S. Alam, "Fog computing for big data analytics in IoT aided smart grid networks," Wireless Personal Communications, vol. 114, no. 4, pp. 3395-3418, 2020.

[23] K. Haseeb, A. Almogren, I. Ud Din, N. Islam, and A. Altameem, "SASC: secure and authentication-based sensor cloud architecture for intelligent internet of things," Sensors, vol. 20, no. 9, p. 2468, 2020. 
[24] M. A. Alanezi, H. R. E. H. Bouchekara, and M. S. Javaid, "Optimizing router placement of indoor wireless sensor networks in smart buildings for IoT applications," Sensors, vol. 20, no. 21, p. 6212, 2020.

[25] T. Han, D. Jiang, Q. Zhao, L. Wang, and K. Yin, “Comparison of random forest, artificial neural networks and support vector machine for intelligent diagnosis of rotating machinery," Transactions of the Institute of Measurement and Control, vol. 40, no. 8, pp. 2681-2693, 2018. 